\title{
Telepresence robots: Encouraging interactive communication between family carers and people with dementia
}

\author{
Wendy Moyle, $\mathrm{PhD}^{*}$ w.moyle@griffith.edu.au \\ Menzies Health Institute Queensland, Griffith University \\ Brisbane, Queensland, 4111, Australia \\ Cindy Jones, $\mathrm{PhD}$ cjones@ bond.edu.au \\ Faulty of Health Sciences \& Medicine, Bond University \\ Gold Coast, Queensland, 4229, Australia \\ Billy Sung, PhD b.sung@curtin.edu.au \\ School of Marketing, Curtin University \\ Perth, WA, 6102, Australia
}

*Correspondence to Professor Wendy Moyle, Menzies Health Institute Queensland, Griffith University, 2.10 Health Sciences (N48), 170 Kessels Road, Nathan, Brisbane, Queensland, 4111, Australia. Tel: +61 73735 5526; Fax: +61 73735 5431; Email: w.moyle@ griffith.edu.au

\section{Acknowledgements}

We thank the family carers, residents, and staff from RSL care Pinjarra Hills, Brisbane for their participation. The study did not receive any financial support from funding agencies in the public, commercial, or not-for-profit sectors. The authors declare no conflicts of interest. 


\title{
Telepresence robots: encouraging interactive communication between family carers and people with dementia
}

\begin{abstract}
Objectives: The aim of the study was to explore the feasibility of using telepresence robots to encourage interactive communication in dementia care, from the perspective of family carers.

Methods: Qualitative findings from semi-structured interviews with six family carers.

Results: Generally, family carers reported a feeling of presence and connectedness when talking to their family member via the telepresence robots. They reported the robots as helping to enhance longer conversations and social connection with their family member.
\end{abstract}

Conclusions: Telepresence may offer potential to encourage social connection, in particular where families are unable to make regular visits to the nursing home.

Word count 99

Key words: dementia, robotics, caregivers, qualitative research, technology 


\section{Introduction}

People with dementia experience memory loss, behavioural and psychological symptoms, such as apathy, agitation, depression, sleep disturbance, impaired reasoning and functioning, and this often results in an inability to socialise and to maintain relationships $[1,2]$. The decline in cognitive and functional ability eventually results in admission to nursing home care where there is reduced opportunities for social connection, leading to loneliness and social isolation $[3,4]$. There are current opinions that telecommunication software such as Skype may help to connect residents, but there is limited evidence in favour of such software, and several studies reporting the technical problems of Skype [5]. Furthermore, a recent study reported the challenges of older people using videoconferencing technologies on iPads [6]. One potential alternative means to connect older people and those with dementia living in nursing home environments with their family and friends is via telepresence, which can help people to feel that are "with the person" [7, 8, 9]. In this study, we sought to explore two types of telepresence robot from the perspective of the family carer.

Telepresence robots are like a 'Skype on wheels' as they are usually freestanding, wheel-based videoconferencing systems that include a web-camera, moveable LCD screen, speaker and microphone. The connection between the telepresence robot and the remote user is established via the internet using specialised software downloaded onto a home computer. The remote user (e.g. family carer) is able to call into the robot and to drive the robot around the nursing home environment, all the while viewing the local user (e.g. person with dementia) via a video display on the robot and via their computer screen. The telepresence robots used in this research are Giraff from Sweden, Camanio Care, AB and VGo from the US, VGo Communications, Inc (Figures 1 and 2). Giraff is almost human height with a large video display, while VGo is the height of a child and its video display, while VGo is the height of a child and with a smaller display compared to Giraff. The height of Giraff can be adjusted into a sitting position when the local user is sitting in a chair to enable the remote user better viewing access. Giraff can also make social gestures by moving the video display up and down (i.e. nodding) and turning side to side. On the other hand, VGo has a streamlined appearance compared to Giraff.

\section{$<<<$ Insert [Figures $1 \& 2]$ here $>>>$}

\section{Methods}

\section{Design}


This paper uses a descriptive qualitative approach [10] to explore the feasibility of using telepresence robots with people with dementia. This approach is useful in this case where information about the phenomenon being experienced is required directly from those experiencing it [11]. Family carers of people living with dementia in long-term care (LTC) were invited to discuss their perceptions of the two telepresence robots; Giraff and VGo following the trial. Ethical approval was provided by (blinded for review) Human Research Ethics Committee (NRS/39/12/HREC).

\section{Participants}

The study involved the following dyads - five persons living with dementia in the care setting and their six family carers ( 2 family carers for 1 resident - see Table 1). A representative from the LTC facility selected participants on the basis of their family's availability, willingness to participate, consent, and the families need for distant communication. All participating residents were diagnosed with mild to moderate dementia, and were considered by staff to be capable of verbal conversation and comprehension, and their eyesight and hearing were adequate for conversations. All family members had access to a Windows computer (i.e., the software only works on the Windows operating system) that enabled installation of the communication software, and sufficient internet speed (i.e., ADSL or equivalent). Written informed consent was obtained from participants (if deemed capable) or next-ofkin at time of enrolment, and participant verbal assent was obtained at each intervention session.

$<<<$ Insert [Table 1] here $>>>$

\section{Intervention}

Families were provided with the respective communication software to connect and operate both Giraff and VGo. A procedure manual was developed by the research team and given to guide the families on how to install the communication software onto their computer, adjust video and audio settings, connect to the robot, drive the robot, and operate different features of the robots. A team member (BS) conducted a practice call with each family member to ensure that they were able to connect to Giraff and VGo situated in the LTC facility, communicate through the robots, adjust field settings, and move the robots around the facility. All dyads participated in the trial of Giraff and VGo, with each trial spanning a five to seven-week period. Dyads were encouraged to make at least five calls on each robot during the trial period, with the duration of each call dependent on the individual situations, without restriction. All calls were separated with at least a 24-hour period and were conducted from the family member's home or workplace and received in the LTC facility. The robot was set up by a research 
assistant before each planned call either in the resident's room or a quiet common area, dependent on the residents' preference and where there was adequate Internet coverage within the facility. The research team was responsible for management of any technical difficulties, such as unforeseen hardware and software problems during the trial.

\section{Data collection and analysis}

Semi-structured interviews were undertaken with the six family carers at the end of the trial. The interview format followed an interview guide developed for the project and sought the carers' perceptions and use of telepresence.

Data were analysed thematically [12]. The study process and qualitative data were reviewed and discussed by all members of the research team throughout the study. During data analysis, the researchers read and reread the data to gain an understanding of the data, coded the data, and revised the concepts accordingly until they agreed on the final themes. Four key themes were identified from the interview transcripts: (i). Enhancing connection; (ii). Familiarity and comfort with technology; (iii). The benefits of mobility; and (iv). Technical difficulties.

\section{Results}

The key themes demonstrate that family members were initially excited to use telepresence as they wanted to connect to their family members on a regular basis. Connection was often challenged by their distance to the facility and work and family commitments. Telepresence offered family members a novel opportunity to encourage regular communication.

\section{Enhancing connection}

Using telepresence was generally described by family carers as giving significant pleasure to the family member and resident over and above a regular telephone call, mainly due to their being able to see each other, their surrounding environments, and enhancing a sense of presence. Telepresence was viewed positively as it was also perceived to encourage longer conversations:

'I think she just really enjoyed it...she just loved being able to see me. Like on one of those occasions it had only been three days since I'd visited her and she was just so excited about being able to see me. We talk on the phone every day. It was just way better. It was great' (P4). 
II was much more chatty than sometimes on the phone, because my mother's speech can be a bit hesitant... Being able to see her somehow I chatted on much more like if I was in the room rather than just on the phone' (P1).

Another family carer who lived overseas highlighted the enhanced quality of connection that telepresence brought to him and his mother:

'Well, if you've got a situation like ours where I'm over the other side of the world...it's far preferable to call via VGo and have that sense of one on one conversation, with an enhanced sense of presence, than it would be for me to actually fly back. To do it by VGo is so superior to telephone ...because you can say things like "Oh, you're looking well" or "You look a bit... whatever". You're actually able to react to the other person and feed that into your conversation' (P3).

In spite of the positive comments about both telepresence robots, most participants felt that Giraff's larger video display size enabled family members to see more of the resident's room, and residents and this was beneficial for facilitating connection when compared to the smaller VGo screen size:

'It was really good being able to actually see where Mum was, get a better view of her room, that sort of thing. I think it was better with Giraff than with VGo' (P5).

I like to be able to see her, to see how she looks. To see if she looks well. To see if her hair is washed and if she's clean and in clean clothes' (P4).

Importantly, telepresence robots were not viewed as a technology that could be used across the trajectory of dementia. One family member described the way that the benefits of the robots were mediated by the level of cognitive functioning of the resident:

I've found it less and less useful throughout the project and that wasn't the technology...that was her. Because there was less and less to talk about because...sadly there is less and less that she can remember' (P5).

\section{Familiarity and comfort with technology}


Where family members were familiar with using video conferencing software this helped them feel comfortable with telepresence:

'I'm used to talking on Skype. I'm used to remote connection for meetings and all sorts of things. So, no, I wasn't concerned. I had a general idea of how it would work' (P3).

Furthermore, having a positive attitude towards technology also assisted participants. Family members perceived that residents' attitude towards the telepresence robots and level of comfort in using this technology tended to improve over time. However, they felt that it may have taken longer for them to become comfortable with the technology than for family members, due to residents having limited previous experience with video conferencing or similar technology:

'I think she got more at ease with it. But she's not as enthusiastic about it as I am. That's possibly a function of age and attitudes to technology. I think the technology worried her. Initially she said to me...I don't see the point.... But, I think she was quite relaxed, more relaxed at it towards the end of it' (P1).

In spite of some concerns, several family members' accounts of residents' attitudes towards telepresence suggested a high level of comfort with the technology and they recognised that telepresence offered additional benefits to the telephone:

'With a telephone, it's something that Mum has had all her life and she quite habitually picks it up and dials. So, she has no problem operating a telephone, but she gets so much pleasure out of the functionality side of the robots and the mobility side of the robots' (P6).

'...I think it was very beneficial for her because she was pleased with herself that she was able to do it, was able to manage it. It was a new experience for her. I think it was stimulating for her' (P3).

'...I think they're fun [the robots] and I think it's a great opportunity for creating interaction and a good experience for Mum...it makes Mum feel special' (P2).

Telepresence was seen to offer an advantage compared to using Skype on a tablet. The robots did not require residents to know how to set up the technology or to manage the software as everything was 
managed remotely by family members. Family members reported that it would be too difficult for residents to use Skype and mentioned that the robots were beneficial because the residents did not need to be concerned with its setup. The resident's cognitive impairment was seen by relatives as impacting on their ability to understand technology or their operation of it:

'I'm assuming that...from her point of view, she wouldn't have to do anything, if it was set up. Because she really isn't capable of doing it... I wouldn't give her an iPad, because she wouldn't be able to do it' (P3).

\section{The benefits of mobility}

For family members who did try moving the telepresence robots, this was mentioned as one of the main benefits over a traditional telephone or Skype call. Family members generally found that it was very easy to use the program to operate the telepresence robots and, overall, found it to be an enjoyable experience to be able to move the telepresence robots around the resident's room, throughout the facility and even into the garden:

'Marvelous enjoyment...Because it was easy and entertaining for Mum and it added scope to the event. It was not just a chat, it becomes an event where you can walk around and I think it's an enjoyment for Mum...I just think it [moving around] enhances the whole experience for Mum. It makes it easy to extend a conversation. We had a chat to that lovely resident, I can't think of his name. Anyhow, we would often just say hi or we would have a chat and it gave mum a chance to talk as well" (P6).

'For me it was great to be able to engage her and get her to take me to places, to take me for a walk. Because I often get concerned that when I'm not there she doesn't walk on her own' (P4).

One family carer reported on the differences in manoeuvrability between Giraff and VGo and on the limitations of their mobility. These included Giraff's wider field-of-view camera making it easier to see where to drive than with VGo, Giraff's movement being less fluid than with VGo due to the smaller size of VGo, and ability to move Giraff's video head up and down, which could not be done with VGo:

'Driving Giraff was surprisingly easy, but then I had been practicing with VGo, so that made a difference. The thing with VGo...you couldn't see as far afield as I could with Giraff, so in that respect driving around, it was probably a little bit easier with Giraff' (P6). 


\section{Technical difficulties}

Although there were many benefits identified to the use of the telepresence robots, there were also technical difficulties that made the experience challenging. These included problems with the internet connection, computer incompatibility (i.e., Mac computers were not compatible), sound distortions, and delay and picture delays. These difficulties were associated with frustration and stress in family carers' experienced with the robots:

'If the internet connection wasn't good it affected the conversation and it also affected the delay in trying to move the machine.' (P4).

Finding an adequate internet connection when outside of the home was often difficult for family. In particular, trying to operate the software within a secure work environment created enormous problems for some family members:

'Well, I think Mum's excitement about it and her enthusiasm I'd have to rate that as a 10. She really looked forward to it. For me on my end I just loved that factor and also loved the opportunity to be able to use it. My stress factor was trying to find an internet - in a busy day trying to find an internet that I could work it from successfully. The difficulty of that is when you're working in businesses they have such tight security on their internet system that they won't let you access it with [telepresence]' (P4).

'...initially I had hoped to use it at home and it's just the internet connection is not good enough at home. Once we moved into the office it was fine - it was a pity we couldn't do it at home because I wanted to do things like show my mother the dog and stuff like that. But, once we got settled in town at my office it was fine' (P1).

Further concerns were raised about the sound. Most participants agreed that there were problems with sound quality on both telepresence robots that seemed, at times, to be related to internet connection. This was of particular concern for one resident whose hearing challenges were identified when she started to use the telepresence robots: 
'...I found that the sound quality on my end wasn't too bad the first time but deteriorated. Until the last time, when I really couldn't hear you or her at all.' (P3).

\section{Discussion}

Family carers in this study reported generally positive experiences with using telepresence and in particular they enjoyed the manoeuvrability of the robots. Being able to see their family member through the video screen was reported to enhance the connection and to encourage a longer conversation. Family carers also enjoyed being able to see that their family member was clean and well-dressed. These were opportunities that are not possible through normal telephone connection without video connection. In fact, the usage of telepresence robotics is supported by the family's involvement in the current research, showing that the feasibility of telepresence robotics are dependent on both family and residents. All residents appeared to benefit from using the robots and eventually they felt comfortable with this new experience. Concerns about a dwindling benefit suggests further research is needed to explore the relationship between telepresence and cognitive severity to understand the point in which the use of such technology is no longer beneficial for people with dementia.

Family carers also reported that telepresence was superior to other communication technologies, such as the telephone and Skype. The large screen, the robot mobility and, importantly, the fact the resident did not have to be involved in the setup of the robot for use made telepresence superior to Skype or FaceTime. However, the current evidence base for Skype remains limited and further studies are needed [5]. A recent integrative review of telepresence robots and their ability to enhance social connectedness reported positive outcomes of using telepresence to connect people with dementia [9]. The review authors reported the main barriers to telepresence were limited experience in using telepresence and they reported similar issues to the current study; technological issues such as software and internet connection problems [9].

In this study, researchers set up the telepresence robot each session to ensure that everything was ready and could check for technical problems prior to the scheduled call. When researchers are not available, the same task could be assigned to a staff member who could also check if the robot has gone back to its dock to be re-charged, and be available to help if the robot, for example, cannot find the resident. As 
the connection is managed remotely, the advantage is that the resident does not have to be involved in setting up of the connection.

The mobility of the telepresence robot encouraged residents and family carers to go for remote walks together into the facility garden and to visit other residents. These extended opportunities for conversations enabled the family carer to get to know other residents, to become aware of the facility and the activities available. In future, telepresence could be used to connect family carers to care staff within the facility and the visiting medical doctor when necessary to seek health information about the resident, or to discuss concerns. Additional features such as vital sign monitoring can allow telepresence to be used outside of the nursing home environment, for example in remote home care applications [13]. This could allow clinicians and caregivers to assist older people in their homes or in assistive living without the need to travel to their locations.

There are several limitations to this research that need to be considered when interpreting the findings. 1) The sample involved only six family carers and, although the interviews provided the opportunity for the family carers to express their perceptions, a larger sample is needed to understand individual characteristics that may influence use of telepresence and the family carers' perceptions. 2)

Telepresence would benefit from being trialled without research staff setting up the robot before the family-resident conversations to ensure the telepresence robot can truly be managed remotely by family carers and facilitated by care staff. 3) We did not ask the people with dementia for their perceptions of the telepresence robots. Future development of telepresence robots needs to include end-users, such as family and people with dementia, in the conceptualisation of the design and the development of the features of the robot to ensure that it is feasible, affordable and an item this population would purchase and use.

\section{Conclusion}

To date there has been limited research undertaken in the use of telepresence with this population and yet, as this research shows, telepresence may offer the potential to socially connect people with dementia living in LTC with family and friends. This has the potential to improve the wellbeing of this population. 
Policy Impact: This research shows telepresence may offer potential within nursing homes to improve communication between family members and residents. Future research may explore the utility of telepresence in reducing loneliness and social isolation in people with dementia in nursing homes. Practice Impact: This research is important in helping researchers, health professionals and developers understand the implications of telepresence for family carers of people with dementia living in nursing homes. 


\section{References}

1. Cerejeira J, Lagarto L, Mukaetova-Ladinska EB. Behavioral and psychological symptoms of dementia. Frontiers in Neurology 2012; 3: 73. doi: 10.3389/fneur.2012.00073

2. Balouch S, Rifaat E, Chen H.L, Tabet N. Social networks and loneliness in people with Alzheimer's disease. International Journal of Geriatric Psychiatric Psychiatry 2019: 34: 666-673.

3. Ellis JM. Psychological transition into a residential care facility: Older people's experiences. Journal of Advanced Nursing 2010; 66: 1159-1168.

4. Moyle W, Venturato L, Griffiths S et al. Factors influencing quality of life for people with dementia: A qualitative perspective. Ageing \& Mental Health 2011; 15: 970-977.

5. Armfield N, Bradford M, Bradford NK. The clinical use of Skype -For which patients, with which problems and in which settings? A snapshot review of the literature. International Journal of Medical Informatics 2015; 10:737-742.

6. Moyle W, Jones C, Murfield J, Liu F. 'For me at 90 its going to be difficult': feasibility of using iPad video-conferencing with older adults in long-term aged care. Aging \& Mental Health 2019; https://www.tandfonline.com/action/showCitFormats?doi=10.1080/13607863.2018.1525605. 7. Moyle W, Jones C, Cooke M, O’Dwyer S, Sung B, Drummond S. Connecting the person with dementia and family: A feasibility study of a telepresence robot. BMC Geriatrics 2014; 14: 7. 8. Nguyen VA, Lu J, Zhao S, Vu DT, Yang H, J DL. Do MN. Immersive telepresence for entertainment and meetings - a practical approach. IEEE Journal of Selected Topics in Signal Processing 2015; 9: 3.

9. Moyle W, Arnautovska U, Ownsworth T, Jones C. Potential of telepresence robots to enhance social connectedness in older adults with dementia: an integrative review of feasibility. International Psychogeriatrics 2017: 29(12):1951-1964.

10. Polit D, \& Beck C.T. (2014). Essentials of nursing research: Appraising evidence for nursing practice $\left(8^{\text {th }}\right.$ ed.). Philadelphia: Wolters Kluters Kluwer.

11. Neergaard M. A., Oleson F, Anderson R, Sondergaard J. (2009). Qualitative description-The poor cousin of health research? BMC Medical Research Methodology, 9, Article 52.

12. Braun V, Clarke V. Using thematic analysis in psychology. Qualitative Research in Psychology 2006; 3: 77-101

13. Laniel S, Letourneau D, Labbe M, Grondin F, Polgar J, Michaud F. Adding navigation, artificial audition and vital sign monitoring capabilities to a telepresence mobile robot for home care application. 
IEEE Explore International Conference on Rehabilitation Robotics (ICORR) 2017; https://doi:10.1109/ICORR.2017.8009347 
Table 1: Participant demographics

\begin{tabular}{|c|c|c|c|}
\hline Demographics & & Resident $(N=5)$ & Family $(N=6)$ \\
\hline \multirow[t]{3}{*}{ Gender } & Male & $1(20 \%)$ & $2(33.3 \%)$ \\
\hline & Female & $4(80 \%)$ & $4(66.7 \%)$ \\
\hline & & 79-89 years & 43-62 years \\
\hline Age & & $\mathrm{M}=85 ; \mathrm{SD}=4.18$ & $\mathrm{M}=54.33 ; \mathrm{SD}=6.31$ \\
\hline Time in Facility & & $\begin{array}{l}1-6 \text { years; } \\
M=2.90 ; S D=2.07\end{array}$ & - \\
\hline \multirow{2}{*}{ Relationship to Resident } & Daughter & & $4(67 \%)$ \\
\hline & Son / Son-in-Law & & $2(33 \%)$ \\
\hline \multirow[t]{3}{*}{ Proximity to Facility } & $\begin{array}{l}\text { Driving Distance } \\
(<5 \text { hours drive })\end{array}$ & - & $3(50 \%)$ \\
\hline & $\begin{array}{l}\text { Flying Distance } \\
\text { (> } 5 \text { hours })\end{array}$ & - & $3(50 \%)$ \\
\hline & Monthly & & $2(33 \%)$ \\
\hline \multirow[t]{3}{*}{ Visitation Frequency } & Quarterly & - & $2(33 \%)$ \\
\hline & Yearly or less & & $2(33 \%)$ \\
\hline & Weekly & & $3(50 \%)$ \\
\hline \multirow[t]{3}{*}{ Phone Call Frequency } & Twice Weekly & - & $2(33 \%)$ \\
\hline & Weekly & & $1(17 \%)$ \\
\hline & Monthly & & $2(33 \%)$ \\
\hline
\end{tabular}


Figure legends

Fig. 1. Giraff

Fig. 2. VGo 